\title{
Flux Motion in YBCO Biepitaxial Josephson Junctions
}

\author{
H. H. Sung \\ Department of Electrical Engineering, Da-Yeh University, ChangHua, Taiwan \\ S. Y. Yang and H. E. Horng \\ Department of Physics, National Taiwan Normal University, Taipei, Taiwan \\ H. C. Yang \\ Department of Physics, National Taiwan University, Taipei, Taiwan
}

\begin{abstract}
We have measured the current-voltage characteristics of overdamped long $\mathrm{YBa}_{2} \mathrm{Cu}_{3} \mathrm{O}_{7}$ biepitaxial Josephson junctions. The velocity-matching step was observed in the current-voltage characteristics with an applied magnetic field. The presence of velocity-matching steps suggests that the biepitaxial Josephson junction is formed around a dielectric-like barrier between superconducting grains. Anomalous subharmonic voltage steps were found under microwave irradiation. The relations between anomaly steps and fluxon motion are discussed.
\end{abstract}

\section{INTRODUCTION}

The magnetic flux motion in a Josephson junction is a subject of increasing practical interest. Since it has been recognized that flux flow three-terminal superconducting devices theoretically can operate at frequencies up to several tens of gigahertz [1], [2]. This makes them attractive for high frequency applications. In a Josephson flux flow transistor, an external magnetic field is applied to the junction plane. The field penetrates into the junction in form of Josephson fluxons, each of them carrying one magnetic quantum $\Phi_{0}$. Ideally, if the bias current is less than the maximum Josephson current $I_{c}$, the phase difference of order parameter on opposite sides of the junction is time independent, and the fluxons are static. When the bias current exceeds $I_{c}$, a Lorentz force will drive fluxons moving along the junction. An electromagnetic (EM) wave also can be generated and propagated along the junction in a slowed velocity, or Swihart velocity [3], $\bar{c}$. In a long junction, there are two main modes of fluxon motion. First is the nonresonant fluxon motion, in which fluxons oscillate between the junction edges. In the second mode, so-called flux flow mode, fluxons are created at one edge of the junction, move along the junction once, and are annihilated at the other edge. When the fluxon velocity $u$ approaches the EM wave phase velocity $\bar{c}$, the current-voltage $(I-V)$ characteristic will show a current step. This step, often referred as the Eck peak [4] or velocitymatching (VM) step, has been observed in both low- $T_{c}$ and high- $T_{c}$ junctions [5].

Manuscript received September 15, 1998.

This work was supported by the National Science Council of ROC under Grant Nos. NSC 87-2112-M-212-001, NSC 87-2112-M-003-001 and NSC 87-2112-M-002-002.
This paper reports our observation of the VM step for YBCO biepitaxial Josephson junctions. From the step voltage, $V_{m}$, the intrinsic capacitance $C$ and the quasiparticle loss $\alpha$ of the junctions can be estimated. By studying the response of these junctions to microwave radiation, we observed the anomaly subharmonic Shapiro steps. These steps are attributed to the synchronous interactions between microwave radiation and fluxon oscillation [6].

\section{EXPERIMENTS}

To fabricate biepitaxial Josephson junctions, a $50 \mathrm{~nm}$ thickness $\mathrm{CeO}_{2}$ buffer was first deposited onto the $\mathrm{MgO}$ [100] substrate by rf sputtering. Part of the $\mathrm{CeO}_{2}$ layer was removed from the surface of substrate by $\mathrm{Ar}$ ion milling, providing a smooth and sharp interface where the grain boundary will be located. Then, a $200 \mathrm{~nm}$-thickness YBCO film was grown on the prepared substrate by rf sputtering. Finally, the YBCO film was patterned by standard photolithography and etched by Ar ion beam.

The electrical measurements were performed using the four-probe method. The current was supplied by a constant current source (Keithley model 220) and the voltage was measured by a nanovoltmeter (Keithley model 182). A magnetic field up to $20 \mathrm{G}$ was applied perpendicular to the

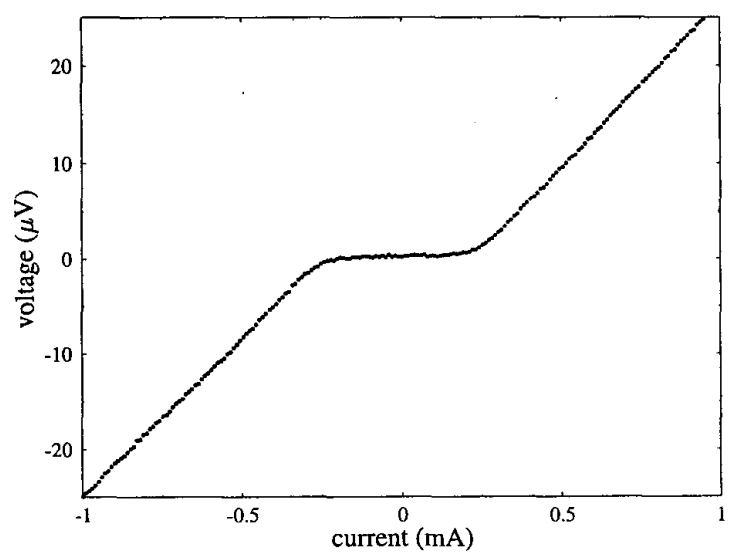

Fig. 1 Current-voltage characteristics of a $30-\mu \mathrm{m}$ YBCO biepitaxial Josephson at $57 \mathrm{~K}$.

$1051-8223 / 99 \$ 10.00$ (C) 1999 IEEE 
sample plane from a calibrated solenoid wrapped around the sample. Microwaves were generated by a microwave generator (GigaTronics model 7200) and were introduced to the junction through a waveguide.

\section{RESULTS AND DISCUSSION}

With no applied magnetic fields or microwave, the I-V curves of our samples exhibit overdamped resistively shunted junction-like features at temperatures up to $80 \mathrm{~K}$. Fig. 1 shows the typical $I-V$ characteristics of a $30-\mu \mathrm{m}$ long YBCO biepitaxial Josephson junction at $57 \mathrm{~K}$. Due to the thermal activated phase slippage, the $I-V$ characteristics show no zero slope at low current and noise rounding curve near the critical current [7].

Using a $1 \mu \mathrm{V}$ criterion, the $I_{c}$ is estimated to be about 330 $\mu \mathrm{A}$, the $I_{c} R_{n}$ is about $12 \mu \mathrm{V}$, and $J_{c}=5.5 \mathrm{kA} / \mathrm{cm}^{2}$ at $T=57 \mathrm{~K}$. However, these parameters can be raised to $2 \mathrm{~mA}, 70 \mu \mathrm{V}$, and $33 \mathrm{kA} / \mathrm{cm}^{2}$ respectively at $25 \mathrm{~K}$. To confirm that the junction is long, we evaluated the Josephson penetration depth

$$
\lambda_{\mathrm{J}}=\left(\Phi_{0} / 2 \pi \mu_{0} J_{c}\left(t+2 \lambda_{\mathrm{L}}\right)\right)^{1 / 2},
$$

where $\mu_{0}$ is the vacuum permeability, $t$ is the thickness of junction barrier, and $\lambda_{\mathrm{L}}$ is London penetration depth. Take a value of $\lambda_{\mathrm{L}}=140 \mathrm{~nm}$ for the YBCO film [8] and $t+2 \lambda_{\mathrm{L}} \approx$ $2 \lambda_{\mathrm{L}}$, thus the $\lambda_{\mathrm{J}}$ is about $4 \mu \mathrm{m}$ and much small than the junction length. The magnetic field dependence of critical current at $25 \mathrm{~K}$ is illustrated in Fig. 2. It can be seen that the $I_{c}(B)$ dependence shows pronounced deviations from the Fraunhofer pattern. The non-Fraunhofer-like $I_{c}(B)$ characteristic has been attributed to crystalline microstructure and $d_{x^{2}-y^{2}}$ symmetry [9], [10]. Since the applied magnetic field is perpendicular to the substrate, the magnetic focus must be considered. Although the curve has lost the regularity of Fraunhofer pattern, the simulation results in [9] show that the separation between the two nearby dips in $I_{c}(B)$

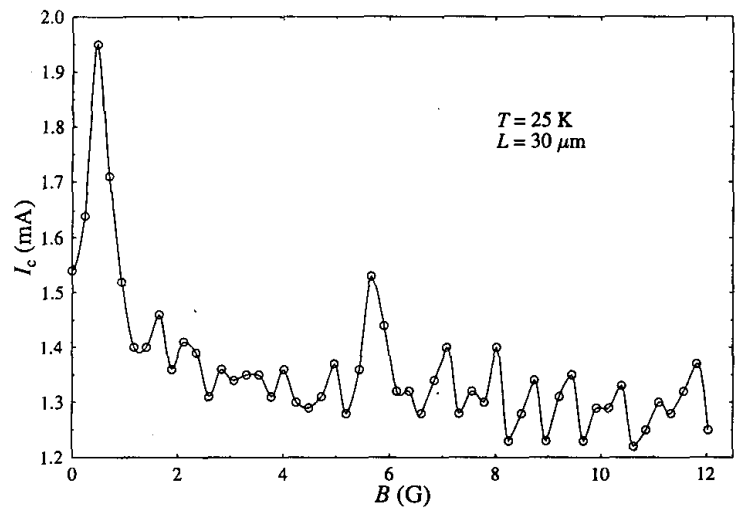

Fig. 2. Critical current as a function of applied magnetic field at $25 \mathrm{~K}$ for the junction with junction length $L=30 \mu \mathrm{m}$. Dots are the experimental data. The lines are guides to the eye. curve is still roughly about one flux quantum in the junction. From Fig. 2, we obtain $0.7 \mathrm{G}$ is approximately corresponding to penetration of one flux quantum into the junction. Thus the flux focus factor $F$ due to the perpendicular applied magnetic field is about 4 .

In order to study flux motion in long YBCO biepitaxial junctions, we applied a magnetic field to a $30-\mu \mathrm{m}$ junction to study $I-V$ characteristics in flux flow state. Fig. 3 (a) shows some results at $57 \mathrm{~K}$. Since the YBCO biepitaxial junctions are overdamped, the VM steps may be obscure in the $I-V$ data Therefore, we plot the excess current $I_{s}$ versus $V$ to reveal the increase of supercurrent, as the dashed lines in Fig. 3 (a) show. Here $I_{s}=I-V / R_{n}$, is the space average dc supercurrent across the junction. Figure 3 (b) shows that the voltage of the increased supercurrent is linearly dependent on the applied field $\mathrm{B}$, and $\mathrm{d} V_{m} / \mathrm{d} B$ is about $22 \mu \mathrm{V} / \mathrm{G}$. Hence we relate these voltages to the VM steps. According to [5], the relation of VM step voltage and applied magnetic filed can be written as

$$
V_{m}=\left(t+2 \lambda_{\mathrm{L}}\right) \bar{c} F B \text {. }
$$

From the value of $\mathrm{d} V_{m} / \mathrm{d} B$, we estimate that the EM wave propagation velocity in YBCO biepitaxial junctions is approximately $2 \times 10^{5} \mathrm{~m} / \mathrm{s}$. This is only about $1 / 50$ of value reported in $\mathrm{YBCO} / \mathrm{YSZ} 0-32^{\circ}$ [001] tilt bicrystal junctions [5]. Since the Swihart velocity is the upper limit of flux flow
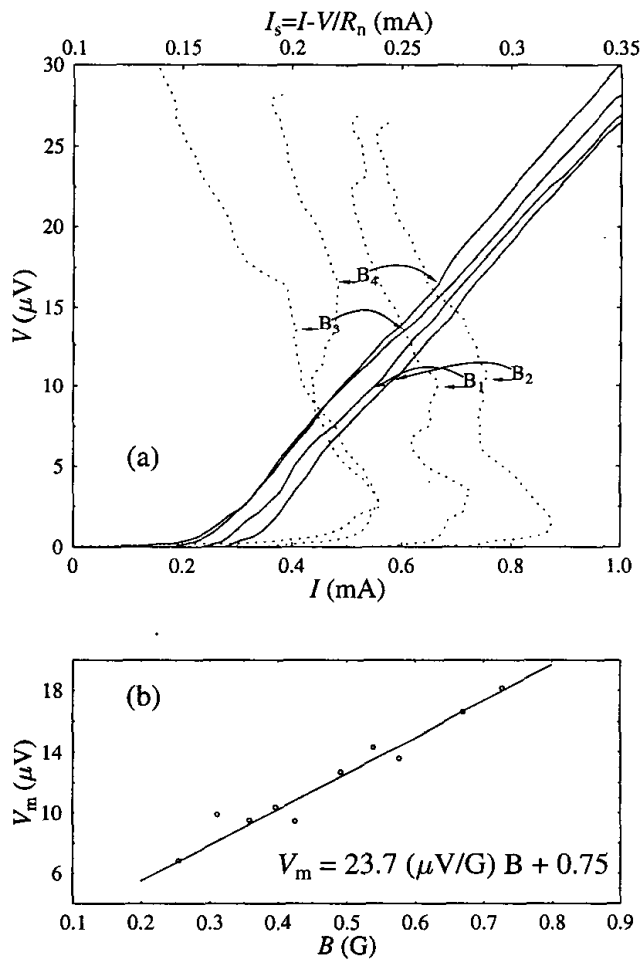

Fig 3. (a) $I-V$ curves of a $30-\mu \mathrm{m}$ junction with different applied magnetic fields $\mathrm{B}_{1}, \mathrm{~B}_{2}, \mathrm{~B}_{3}, \mathrm{~B}_{4}$ at $57 \mathrm{~K}$. The average supercurrent $I_{5}$ vs. $V$ curves are shown as dash lines. The arrows indicate the $V_{\mathrm{m}}$ voltages where flux flow resonances occur. $B_{1}=0.31 \mathrm{G}, B_{2}=0.40 \mathrm{G}, B_{3}=0.58 \mathrm{G}, B_{4}=0.67 \mathrm{G}$. (b) VM step voltage $V_{\mathrm{m}}$ versus applied magnetic field $\mathrm{B}$ of the $30-\mu \mathrm{m}$ junction. The solid line is the linear fit. 


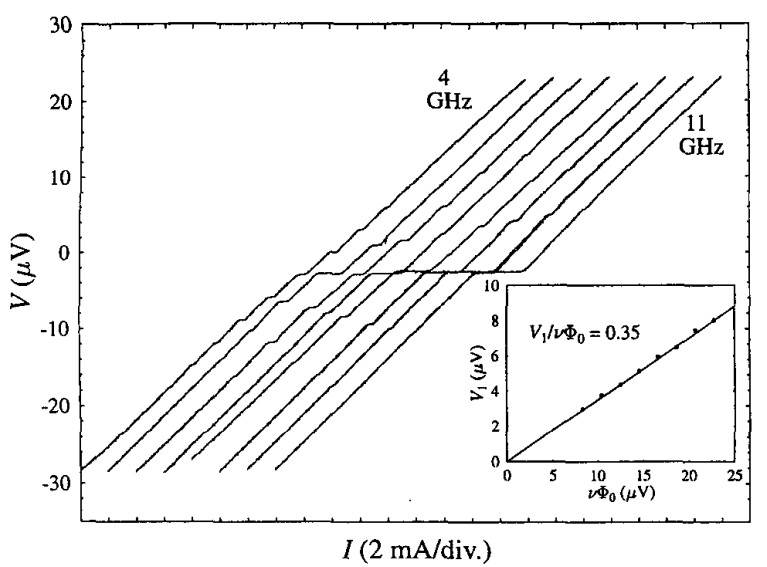

Fig. $4 I-V$ curves of a $10-\mu \mathrm{m}$ junction irradiated with different microwave frequencies at $67 \mathrm{~K}$. The inset shows the first step voltage as a function of production of microwave frequency and flux quantum $\vee \Phi_{0}$. The solid line shows linear fitting curve.

velocity, the maximum operation frequency of Josephson flux flow devices is about $20 \mathrm{GHz}$.

Moreover, the Swihart velocity can be established as

$$
\bar{c}=c\left(\frac{\varepsilon_{0}}{C\left(2 \lambda_{\mathrm{L}}+t\right)}\right)^{1 / 2}
$$

where $c$ is the light velocity in vacuum, $\varepsilon_{0}$ is the permittivity of vacuum, and $C$ is the intrinsic capacitance per unit area of the junction. Accordingly, we obtain $C=70 \mathrm{pF} / \mu \mathrm{m}^{2}$, and a junction capacitance of $420 \mathrm{pF}$. Since our junctions show no hysteresis, this corresponds to a Stewart-McCumber parameter $\beta_{c}$ of less than one. Indeed, the $\beta_{c}$ we calculated is $0.5<1$, and is consistent with non-hysteresis $I-V$ curves. Furthermore, the quasiparticle loss $\alpha=\left(1 / I_{c} R_{n}\right)\left(J_{c} \Phi_{0} / 2 \pi C\right)^{1 / 2} \approx 1.3$ at $57 \mathrm{~K}$. This confirms that the EM wave can propagate in the junction, and flux flow can be sustained.

Finally, in Fig. 4, we report the $I-V$ characteristics of a 10 $\mu \mathrm{m}$ junction irradiated with different microwave frequencies at $67 \mathrm{~K}$. The $\mathrm{I}-\mathrm{V}$ curves show some constant voltage steps, such as the well known Shapiro steps. However, the value of the first step voltage $V_{1}$ divided by the product of microwave frequency and flux quantum $v \Phi_{0}$ is 0.35 , as shown in the inset of Fig. 4. There are several mechanisms to explain subharmonic steps. Non-integral steps have been observed in the YBCO biepitaxial junctions [11], and interpreted as the effect of parallel junctions. However, parallel junctions can describe the $1 / 2,1 / 3$ steps, and this is not the case here. Twodimensional Josephson arrays can cause subharmonic steps when the applied field is such that the number of flux quanta per unit cell is not an integer [12]. However, the applied magnetic field does not affect the step voltages in our sample. Other possibilities are the nonsinusoidal current-phase relation (CPR) [13], [14] and the effect of flux motion in a long junction. The nonsinusoidal CPR for $45^{\circ}$ grain boundary YBCO junctions was observed by Il'ichev et al. [15]. Thus, we cannot rule out the prospect in our YBCO biepitaxial junctions. On the other hand, since we have observed flux flow resonance in the $30-\mu \mathrm{m}$ junction, it is reasonable to examine the effect of flux motion.

For microwave radiation at powers less than the threshold for flux flow, constant voltage steps can be induced at [6]

$$
V_{n / m}=(n / m)\left(\nu \Phi_{0}\right)
$$

These voltage steps are due to the synchronous coupling that occurs when the fluxon couples to the $n$th harmonic of the incident radiation for every $m$ trips across the junction. In other words, the condition for which $V_{n / m}$ appears is $v_{\Phi}=$ $(n / m) v$, where $v_{\Phi}$ is the frequency of fluxon oscillation in the junction. If the anomalous subharmonic voltage steps we observed are caused by interaction between microwave radiation and fluxon oscillation in the long junction, then the average velocity of flux motion $\bar{u}$ can be calculated using $\bar{u}=L v_{\Phi}$, where $L$ is the junction length. With the ratio $n / m=$ 0.35 , we obtained the $\bar{u}=2.1 \times 10^{4} \mathrm{~m} / \mathrm{s}$ for a YBCO biepitaxial junction at $6 \mathrm{GHz}$ microwave irradiation. Although the average velocity $\bar{u}$ is consistent with the upper limit of fluxon velocity in the above discussion, additional experiments are necessary, such as to determine the dependence between junction length and step voltage.

In conclusion, we have studied the $I-V$ characteristics of YBCO biepitaxial Josephson junctions under magnetic fields and microwave irradiation. The VM steps were observed in the $30-\mu \mathrm{m}$ junctions at $57 \mathrm{~K}$. This suggests that EM waves can propagate along the junction, and the junction capacitance cannot be neglected. However, the StewartMcCumber parameter of the junction is about 0.5 , which still satisfies the non-hysteresis $I-V$ characteristics shown in our junctions. Finally, we observed the anomalous subharmonic voltage steps in the microwave irradiated $I-V$ curves. The anomaly cannot be attributed to the effects of parallel junctions or $2 \mathrm{D}$ junction arrays. However, the other two possible mechanisms, flux motion in a long junction and nonsinusoidal $\mathrm{CPR}$, require further experiments in order to determine which is the cause.

\section{REFERENCES}

[1] J. Mannhart, "High- $T_{\mathrm{c}}$ transistors," Supercond. Sci. Technol., vol. 9, pp. 49-67, 1996.

[2] J. E. Nordman, "Superconductive amplifying devices using fluxon dynamics," Supercond. Sci. Technol., vol. 8, pp. 681-699, 1995.

[3] J. C. Swihart, "Field Solution for a Thin-Film Superconducting Strip Transmission Line," J. Appl. Phys. vol. 32, pp. 461-469, 1961.

[4] R. E. Eck, D. J. Scalapino, and B. N. Taylor, "SELF-DECTION OF THE ac JOSEPHSON CURRENT," Phys. Rev. Lett., vol. 13, pp. 15-18, 1964.

[5] Y. M. Zhang, D. Winkler, P.- $\AA$. Nilsson, and T. Claeson, "Josephson fluxflow resonance in overdamped long $\mathrm{YBa}_{2} \mathrm{Cu}_{3} \mathrm{O}_{7}$ grain-boundary junctions," Phys. Rev. B, vol. 51, pp. 8684-8687, 1995.

[6] M. Scheuermann, J. T. Chen, and Jhy-Juin Chang, "Interaction between microwaves and a single vortex in a long Josephson tunnel junction," $J$. 
Appl. Phys., vol. 54, pp. 3286-3290, 1983.

[7] Vinay Ambegaokar and B. I. Halperin, "VOLTAGE DUE TO THERMAL NOISE IN THE dc JOSEPHSON EFFECT," Phys. Rev. Lett. vol. 22, pp. 486-1366, 1963.

[8] M. C. Nuss, K. W. Goossen, P. M. Mankiewich, and M. L. O'Malley, "Terahertz surface impedance of thin $\mathrm{YBa}_{2} \mathrm{Cu}_{3} \mathrm{O}_{7}$ superconducting films," Appl. Phys. Lett. vol. 58, pp. 2561-2563, 1991.

[9] C. A. Copetti, F. Rüders, B. Oelze, Ch. Buchal, B. Kabius, and J. W. Seo, "Electrical properties of $45^{\circ}$ grain boundaries of epitaxial YBaCuO, dominated by crystalline microstructure and d-wave-symmetry," Physica C, vol. 253, pp. 63-70, 1995

[10] H. Hilgenkamp, J. Mannhart, and B. Mayer, "Implications of $d_{x^{2}-y^{2}}$ symmetry and faceting for the transport properties of grain boundaries in high- $T_{\mathrm{c}}$ superconductors," Phys. Rev. B, vol. 53, pp. 14586-14593, 1996

[11] E. A. Early, A. F. Clark, and K. Char, "Half-integral constant voltage steps in high- $T_{\mathrm{c}}$ grain boundary junctions," Appl. Phys. Lett., vol. 62, pp. 3357-3359, 1993.

[12] S. F. Benz, M. S. Rzchowski, M. Tinkham, and C. J. Lobb, "Fractional Giant Shapiro Steps and Spatially Correlated Phase Motion in 2D Josephson Arrays," Phys. Rev. Lett., vol. 64, pp.693-696, 1990

[13] D. B. Sullivan, Robert L. Peterson, V. E. Kose, and J. E. Zimmerman "Generation of Harmonics and Subharmonics of the Josephson Oscillation," J. Appl. Phys., vol. 41, pp.4865-4873, 1970.

[14] K. H. Gundlach and J. Kadlec, "ac Josephson-Like Effect with LinearPeriodic Current-Phase Relation," J. Low Temp. Phys., vol. 26, pp. 603$612,1977$.

[15] E. Il'ichev, V. Zakosarenko, R. P. J. IJsselsteijn, V. Schultze, H.-G Meyer, and H. E. Hoenig, "Nonsinusoidal Current-Phase Relationship of Grain Boundary Josephson Junctions in High- $T_{\mathrm{c}}$ Superconductors," Phys. Rev. Lett., vol. 81, pp. 894-897, 1998 